\title{
The Design of personalized glass frame Based on Reverse Engineering and 3D Printing
}

\author{
Jie Liü, Guanping Dong ${ }^{\mathrm{b}}$ and Yanbin Fan ${ }^{\mathrm{c}}$
}

The Mechatronics and Eletronic Engineering School, Foshan University, Foshan 528000, China

ajie.liu.scut@gmail.com, bdonggp@163.com, ${ }^{c}$ berlin1989@163.com

Keywords: 3D Printing; Reverse Engineering; Personalization; Rapid Manufacturing; Glass Frame

\begin{abstract}
With the development of modern society, private custom glasses has become a trend for future development. Traditional handmade time about two weeks, and processing fee is also high. But if you use 3D printing processing, it will reduce the processing time and reduce the cost of processing, and more precise. In this paper introduced a 3D printing manufacturing methods of personalized glass frame.
\end{abstract}

\section{Introduction}

$3 \mathrm{D}$ printing is also called rapid prototyping manufacturing, which is under computer control, using different methods by the principle of discrete, the accumulation of material accumulation, the forming and manufacturing technology of finished parts[1].Help people vision correction is the preoccupation in the traditional glasses manufacturing, but now, with the improvement of people's living standard, more and more people wearing glasses is not considered it's help for the eyesight but as necessities of life, which can show their personality and charm[2]. Some companies have begun to appear in the society, which can be customized according to customer requirements the head size of the glasses with personal characteristics[3]. the high cost of production time is longer that lead to personalized glasses hard to popularize. in America, which has the world's largest 3D printer manufacturers, that make 3D printing in the United States had a very good development environment and infrastructure. therefore, if the 3D printing technology is applied to the production of personalized glasses, the processing cost and reduce the processing time will reduce. It's will make it easier to develop and popularize[4-10].

\section{The design data acquisition of glass frame}

We need to get the following data before design the glass frame, the width of the glasses, ear the distance to the eye and the bridge of the nose width, height and angle of data. According to the data drawing, Glass frame design involves the optical center, So we also need carry on the optical center design. The location determined by glasses wear position, general in the glass frame $1 / 2 \sim 2 / 3$ ideal position.

\section{Manufacturing principle of glass frame 3D printing}

This personalized glass frame of 3D printing with the introduction of Fused Deposition Modeling (FDM) to manufacture. The principle of the manufacturing process is that by putting a filamentous heating thermoplastic, and then squeeze out from the printer nozzle. Layer upon layer overlay fabricates according to the desired trajectory. Materials piled up at a fixed speed, The workbench will decline when accomplished each layer, over and over again. Finally realizes the glass frame deposit forming. The key technology of FDM is keeping the temperature of half flow molding material above the melting point. At the same time, the thickness of each layer usually is $0.25 \sim 0.50 \mathrm{~mm}$. The advantage of FDM is high material utilization, low cost, simple process. but the disadvantage is precision low, complex components are not easy to manufacture, the cantilever needs to support, poor 
surface quality. The manufacturing process of 3D printing suitable for medium and small parts, not the large part.

\section{The design of glass frame}

4.1 Data processing and the design of the parts

The production of ordinary glass frame is a standard to follow. Because the center frame size and distance apart is exactly equal to the length of two eye pupil center, When the lens production, we need according to the calculated eccentricity of the single eye pupil to adjust the lens. According to the calculated eccentricity of the eye pupil to adjust the lens, During design, we can direct the eye pupil center distance as the frame geometry size, so we can save the amount owing to the eccentricity of the single eye pupil, lead to the processing of the lens.

\subsection{Glass frame foot length calculation}

When overlooking the spectacle frame, we can see its leg and mirrors have an opening Angle and the Angle between circle. we design Opening Angle and external opening Angle must as far as possible in conformity with the characteristics of the wearer's head. Among them, the opening Angle depends on the size of the ear width and the ratio of head width.

\subsection{D printing manufacture of glass frame}

This study used printer is Dimension 3D printers series of Elite models, it's the top models in this series. Its biggest print size is $8 * 8 * 12$ inches, the precision of the equipment can reach $0.08 \mathrm{~mm}$, It also equipped with the latest type support system. and the equipment use high strength materials for ABS Plus, which strength can reach $140 \%$ of the original ABS material, The support material in print is completed by a specific solution to clean up, So even if is extremely complex and fine product, the internal structure of finished product. The whole process also can be seen clearly without any manual processing. To finish the work mainly do the following 4 steps.

- File format conversion. First of all, convert 3D drawings to the STL format, and then, use software of magics 13.0 will repair the file, finally, put the files in the USB flash drive, and then copy to the computers connected to the 3D printer. The software is working for repair as shown in Fig. 1

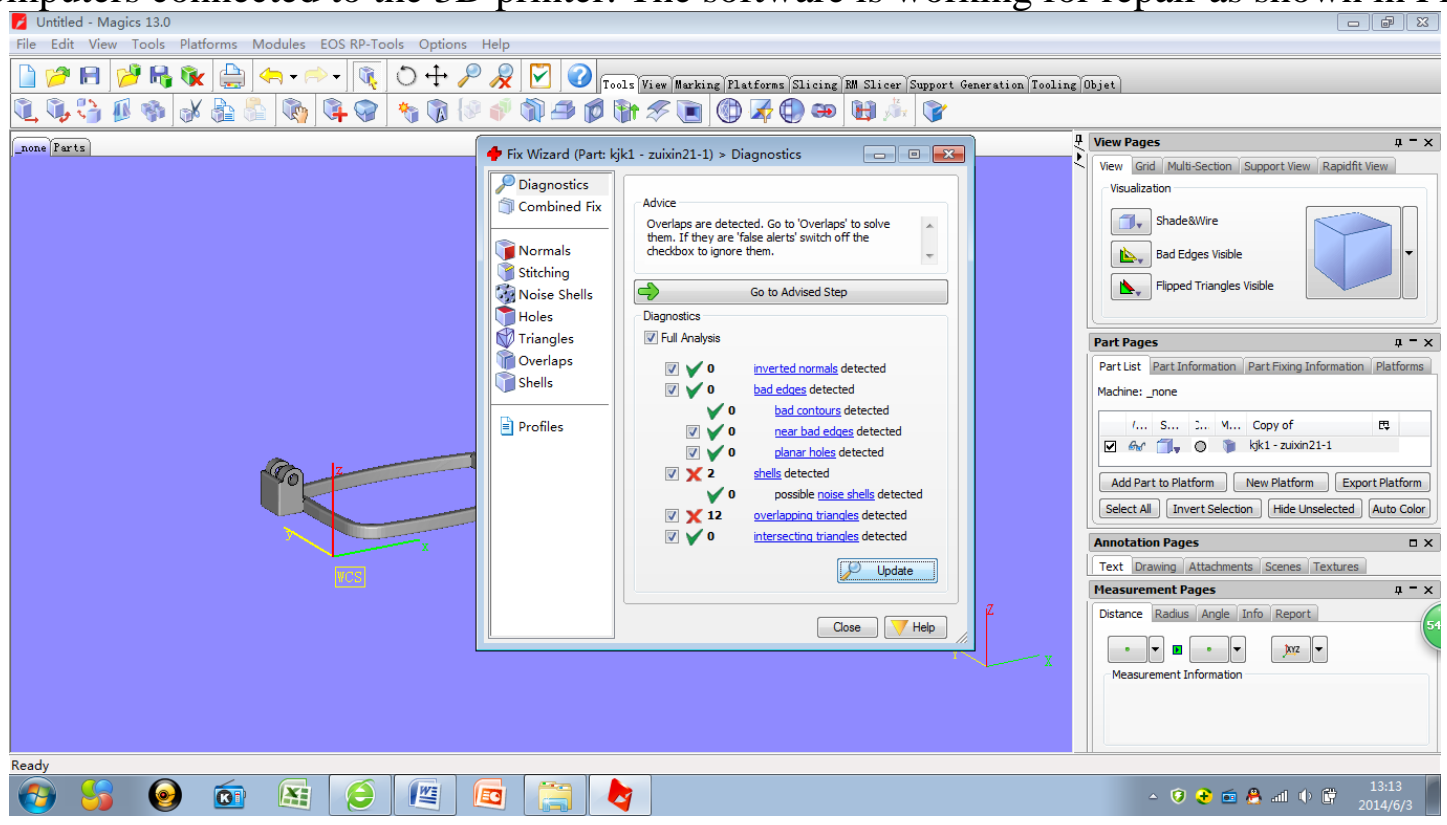

Fig. 1 Magics software is working for repair

-Import STL file. In this step, open the software import printing file, components of the three-dimensional figure appeared on the software. The print direction of choice model, this step is very important, and then enter the next step. 
- Model simulation of $3 D$ printing. Before the model entity processing, to ensure the accurate of the print, need to advance simulation print on a 3D printer. In this steep, we need to determine the part's data of position, material strength. After completing the above operation can start the processing. $3 \mathrm{D}$ printing process is working is working as shown in Fig. 2

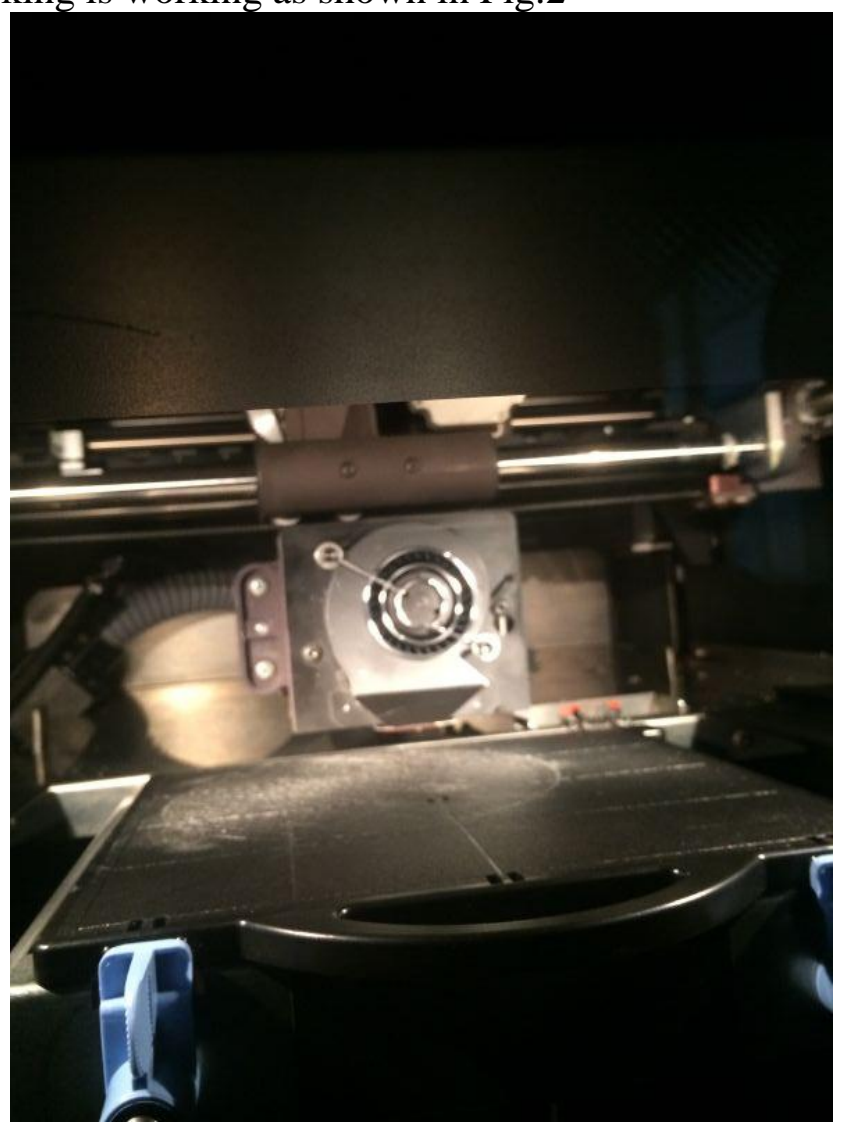

Fig. 2 3D printing process is working

- The entity processing manufacturing. Close to $70 \%$ of the glass frame is made of ABS material. The whole process is a physical change, so the size will not warp shrinkage after forming. Processing completed glass frame by 3D printing as shown in Fig. 3

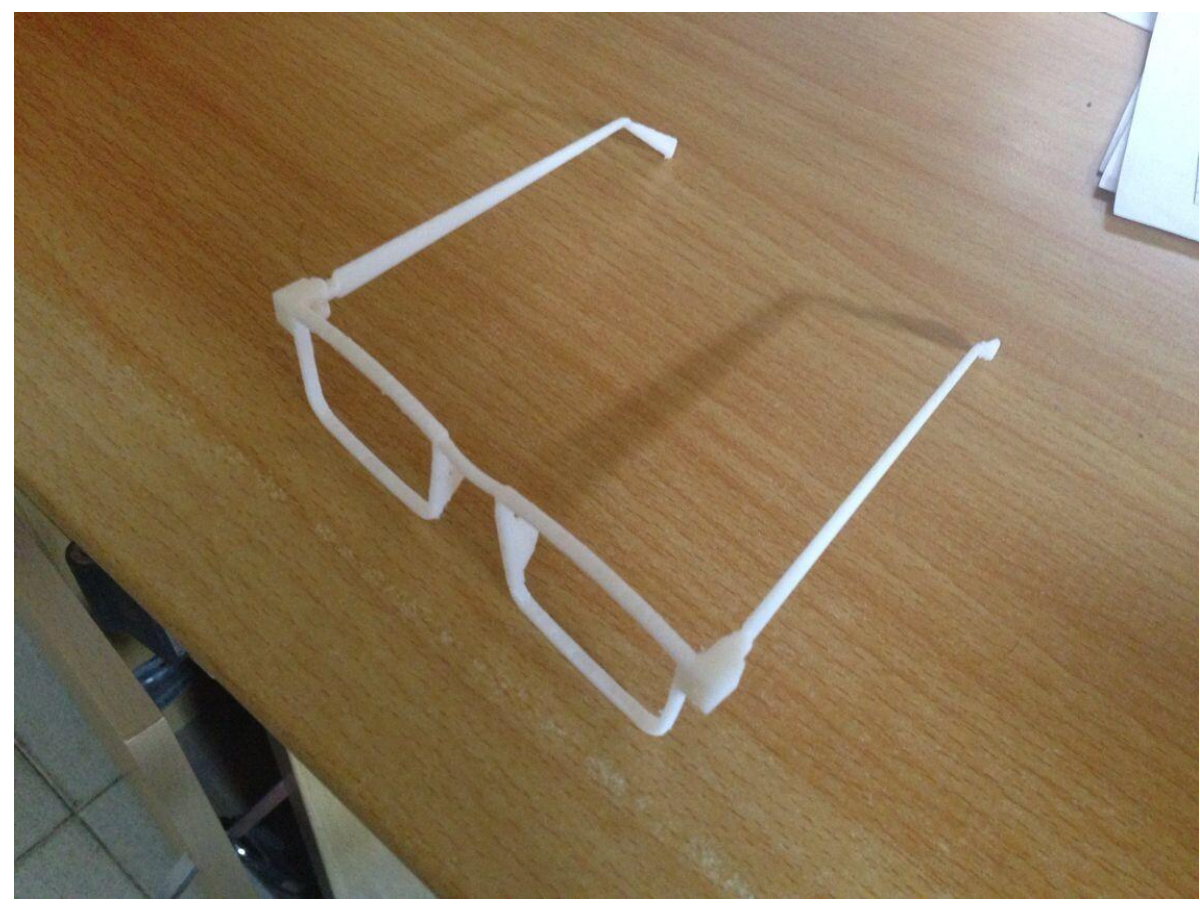

Fig. 3 Processing completed glass frame by 3D printing 


\section{Conclusions}

This is a show of 3D printing technology can be applied to People's Daily life. Although this design is successfully manufacture, but in the whole process have found some problems, such as measurement data is not accurate enough. We're going to get more accurate data if use the scanning instrument for data collection, but high cost. In this case, this is not conducive to realize the industrialization promotion. At the same time, 3D printing technology have defects on the bending strength of the product is poorer, inadequate accuracy. These defects will continue to improve as 3D printing technology improvement, the prospect of the industry will get better.

\section{Acknowledgements}

This work was financially supported by the Natural Science Foundation of Guangdong Province (2014A030310301), the Science and Technology Planning Project of Guangdong Province (2014A0 10106002), the Medical Science and Technology Research Project of Foshan (20141020149), the ma jor scientific and technological projects of Foshan (2013Z21001) and the Scientific Research Startup Foundation of Foshan University.

\section{References}

[1]F.Feng: New Technology \& New Products of China Vol. 16 (2015), p. 61

[2] T.Wang: Modern Manufacturing Engineering, Vol.02(2015), p. 89

[3] Z.H.Wang, Y.F.Li and M.Y.Zhang: Economic Review Vol. 01 (2013), p. 90

[4] X.Z.Guo, Q.W.Deng:National Defense Science \& Technology Vol.36 (2015), p. 36

[5] C.Ge, G.Y.Fu, C.F.Sun and L.Gong: New Technology \& New Process, Vol. 04 (2006), p. 26

[6] Y.Q, Yang, Y.Liuand and C.H.Song: Mechanical \& Electrical Engineering Technology, Vol. 04 (2013), p. 01

[7] J.Liu, Y.Q.Yang and C.H.Song: Journal of South China University of Technology (Natural Science Edition) Vol. 39 (2011), p. 13

[8] Y.Q.Yang, X.R.He, W.H.Wu, H.W.Ding, D.Wang, T.T.Sun, W.H.Huang: Chinese Journal of Lasers Vol.36 (2009), p. 246

[9] Y.Q.Yang, J.B.Lu, Z.Y.Luo, D.Wang: Rapid Prototyping Journal Vol.18 (2012), p. 482

[10]J.H.Xu, M.Zhang: Packaging Engineering, Vol. 06 (2004), p. 131 